\title{
Theoretical Analysis of Nanogenerators with Aligned Nanorods for Piezoelectric Energy Harvesting
}

\author{
Jundong Song, ${ }^{1}$ Tomoaki Yamada, ${ }^{1,2^{*}}$ Masahito Yoshino, ${ }^{1}$ and Takanori Nagasaki ${ }^{1}$ \\ ${ }^{1}$ Department of Energy Engineering, Nagoya University, Nagoya 464-8603, Japan \\ ${ }^{2}$ PRESTO, Japan Science and Technology Agency, Kawaguchi 332-0012, Japan
}

(Received October 3, 2018; accepted January 30, 2019)

Keywords: piezoelectric energy harvesting, nanorods, finite-element simulation

Piezoelectric energy harvesters are expected to power trillions of miniaturized sensors. We theoretically analyze nanogenerators with aligned piezoelectric nanorods using formulaic solutions and finite-element simulation using COMSOL Multiphysics in order to explore the possibility of achieving excellent output performance. It is shown that for the same piezoelectric materials, using a rod-shaped unit will increase the piezoelectric constant $d_{33}$ by $33 \%$ compared with using a film, owing to less substrate clamping. In addition, when normal force is applied, the stress is concentrated in the nanorods. Therefore, in the case of applying normal force, the aligned nanorod array can generate an output power one or more orders of magnitude higher than films within the same volume. This type of nanogenerator will play an important role in the integration with miniaturized sensors in the off-resonant mode.

\section{Introduction}

We used to dream of a world where inanimate objects become animate and even have feelings. Now this dream may come true when such objects are embedded with wireless sensor nodes and linked to the Internet; we call this the Internet of Things (IoT). ${ }^{(1,2)}$ For this system, energy harvesting, a promising technology that scavenges ambient energy such as vibration, heat, or light to generate power, will play a very important role as a renewable power source for trillions of wireless sensors. Unlike batteries that require frequent detaching or changing, energy harvesting is effective over a long period and causes negligible pollution. Furthermore, energy-harvesting devices can be processed on a microscale or even less and easily integrated within the sensors, reducing the cost of both production and operation. ${ }^{(3)}$

As one of the most effective ways to harvest energy, piezoelectric energy harvesting (PEH) has attracted great interest because vibration sources are ubiquitous. Indeed, the output power of PEH devices is far out of reach of giant machines but is expected to be at a milliwatt level or higher after further investigation. According to the expression of the available energy $E_{a}$, which is proportional to the output power, the figure of merit (FOM) $d^{2} / \varepsilon$, applied stress $T$, and volume $U$ are found to be the influential factors, as shown in ${ }^{(4)}$

*Corresponding author: e-mail: t-yamada@energy.nagoya-u.ac.jp

https://doi.org/10.18494/SAM.2019.2149 


$$
E_{a}=\frac{1}{2} C V^{2}=\frac{d^{2}}{2 \varepsilon} T^{2} U
$$

where $C, V, d$, and $\varepsilon$ are the capacitance, voltage, piezoelectric constant, and permittivity, respectively. FOM is a property inherent to the material itself. The main obstacle to achieving a large FOM is that the piezoelectric constant and permittivity strongly correlate with each other. As shown in Table 1, FOM values of different materials are similar, whereas $d_{33}$ varies largely.

Another way to increase the output power is to induce a large $T$ by optimizing the design of PEH devices. One of the effective solutions is to use a cantilever at resonance. However, it becomes more difficult for miniaturized devices to resonate at a frequency lower than $300 \mathrm{~Hz}$ for ambient vibrations, especially with the traditional design of ceramic layers with metal or silicon substrates. In this case, flexible PEH devices are taken into consideration with the expectation of a low resonant frequency and crack-free operation as frequently reported. ${ }^{(6-8)}$ The applicability of piezoelectric polymers such as polyvinylidene fluoride (PVDF) and many polymer-based piezoelectric composites is also shown.

Instead of using resonance, a type of PEH structure with nanorods aligned in the $d_{33}$ mode upon applying normal force can be another effective solution ${ }^{(9-11)}$ and is investigated in this study. The evaluation of the output performance by theoretical analysis showed that the nanorod structure provides advantages in inducing a large stress as well as improving FOM, compared with piezoelectric films. With respect to miniaturized devices, it is possible for nanorod arrays to generate a higher output power than the traditional cantilever and meet the off-resonant applications.

\section{Theoretical Analysis}

The output power of the aligned nanorod array is derived from calculated results based on the piezoelectric equation as well as related equations of the electric circuit. Sinusoidal vibration $F=F_{m} \sin (2 \pi f t)$ is applied along the axial direction of nanorods, namely, the direction normal to the substrate, causing the $d_{33}$ working mode. The output power can be calculated when the nanorod array is connected to an external resistance $R$ (Fig. 1). Then the nanorod array is modeled and simulated using the finite-element simulation software COMSOL Multiphysics $5.3 a$ to validate the calculated prediction.

Table 1

$d_{33}$ and FOM values of various piezoelectric materials. ${ }^{(5)}$

\begin{tabular}{lcc}
\hline Materials & $d_{33}(\mathrm{pC} / \mathrm{N})$ & $\mathrm{FOM}_{33}=\frac{d_{33}^{2}}{\varepsilon}\left(10^{-12} \mathrm{~Pa}^{-1}\right)$ \\
\hline $\mathrm{Pb}(\mathrm{Zr}, \mathrm{Ti}) \mathrm{O}_{3}$ & $225-590$ & $5.4-14.4$ \\
$\mathrm{BaTiO}_{3}$ & $125-260$ & $\sim 5$ \\
$(\mathrm{~K}, \mathrm{Na}) \mathrm{NbO}_{3}$ & $104-490$ & $10-14$ \\
$\mathrm{PVDF}$ & $-(20-24)$ & $4-8$ \\
\hline
\end{tabular}




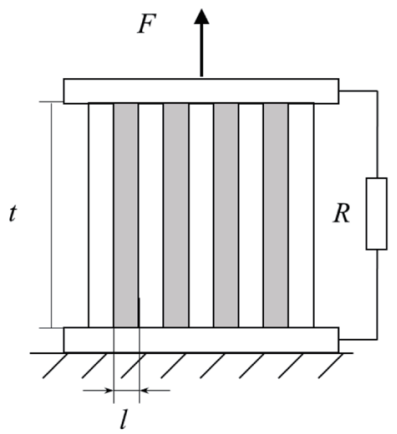

(a)

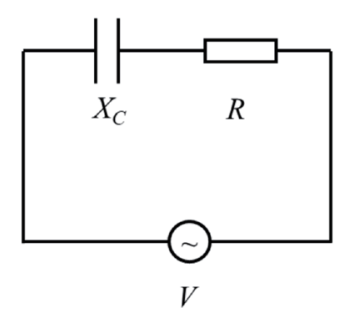

(b)

Fig. 1. (a) Schematic model of the nanorod array connected to resistance. A rod unit is treated as a cuboid with $t$ and $l$ representing the height and width, respectively. (b) Equivalent circuit where the nanorod array is treated as a combination of the energy source $V$ and capacity whose impedance is $X_{c}$.

\subsection{Formulaic solutions}

For a piezoelectric bulk with electrodes at the top and bottom, the output voltage $V_{3}$ under the open-circuit condition can be expressed as

$$
V_{3}=E_{3} t=-\frac{t}{\varepsilon} d_{33} T_{3},
$$

where $E_{3}$ stands for the electric field, and $d$ and $T$ are described with Voigt notation. In general, when the tensile stress is applied along the longitudinal 3-direction, the piezoelectric bulk will shrink in the transversal 1,2-direction while elongating along the 3-direction [Fig. 2(a)]. However, for a piezoelectric film clamped by the substrate, deformation in the 1,2-direction is suppressed, meaning that $T_{1}$ and $T_{2}$ do not equal zero [Fig. 2(b)]. Thus, we have

$$
\begin{gathered}
S_{1}=s_{11} T_{1}+s_{12} T_{2}+s_{13} T_{3}+d_{31} E_{3}=0, \\
D_{3}=d_{31} T_{1}+d_{32} T_{2}+d_{33} T_{3}+\varepsilon E_{3},
\end{gathered}
$$

where $S_{1}$ stands for the strain. If $T_{1}=T_{2}$ and $d_{31}=d_{32}$, Eqs. (3) and (4) can be deduced as

$$
d_{33}^{\prime}=d_{33}+2 d_{31} \frac{d_{31} d_{33}-\varepsilon s_{13}}{\varepsilon\left(s_{11}+s_{12}\right)-2 d_{31}^{2}}
$$

where $d_{33}^{\prime}$ represents the piezoelectric constant of the clamped films. It is obvious that $d_{33}^{\prime}$ is lower than $d_{33}$. This clamping effect will be reduced if the piezoelectric unit has a large aspect ratio $t / l$, such as that of nanorods. Therefore, the nanorod array will be expected to show a larger output voltage than the film. 


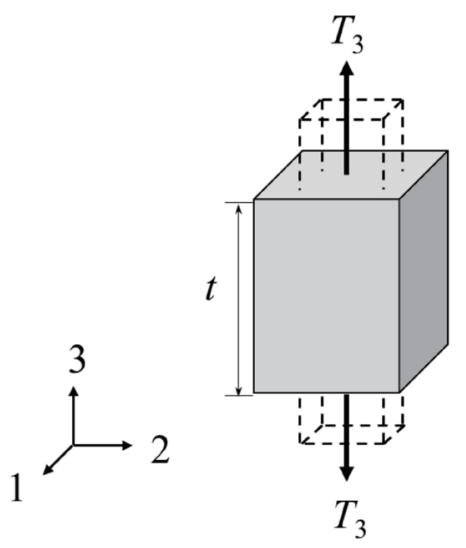

(a)

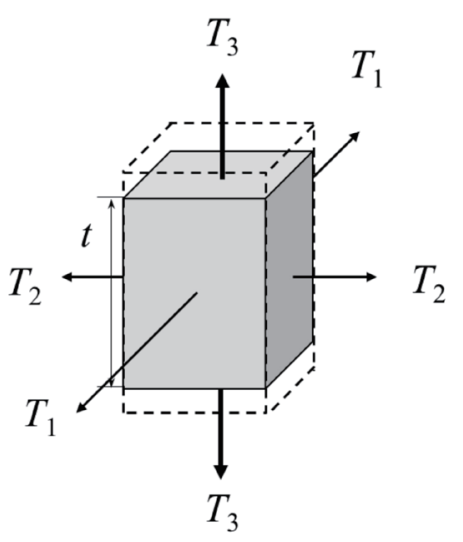

(b)

Fig. 2. Deformation of piezoelectric bulk (a) and clamped piezoelectric film (b) upon applying stress $T_{3}$.

In addition, the output voltage of the nanorod array will be even more enhanced than that of the film of the same total volume. Given a vibration with a constant amplitude $F_{m}$ uniformly applied to the top electrode surface, the stress applied to the nanorods becomes larger than that to the film owing to the lower density of the piezoelectric material. Therefore, the voltage ratio between the nanorod array and the film can be expressed as

$$
\frac{V}{V_{0}}=\frac{d_{33}}{d_{33}^{\prime}} \frac{A_{0}}{A}
$$

where the subscript " 0 " represents the film and $A$ stands for the contact area between piezoelectrics and electrodes. According to Eq. (7) below, deduced from Ohm's law, the ratio of output power within $V_{0}$ can be deduced as Eq. (8).

$$
\begin{gathered}
P=\left|\frac{1}{2}\left(\frac{V_{m}}{-j X_{C}+R}\right)^{2} R\right| \leq \frac{V_{m}^{2}}{4 X_{C}}, \\
\frac{P}{P_{0}}=\frac{A_{0}}{A}\left(\frac{d_{33}}{d_{33}^{\prime}}\right)^{2} .
\end{gathered}
$$

Here, $P$ is maximum at $R=X_{c}$. Equations (6) and (8) indicate that the output will be heightened as the area (also referred to as the density) of nanorods is reduced, even if the capacitance becomes smaller. The result also emphasizes that the stress applied to the piezoelectric material has a larger effect on the output power owing to a quadratic relationship. 


\subsection{Modeling and simulation}

The above predictions from formulaic solutions are validated in COMSOL. Figure 3 shows the boundary conditions of the nanorods for the $d_{33}$ mode and the conventional cantilever for the $d_{31}$ mode. Rigid boundaries without moving in the $x$ - and $y$-directions are set at the top and bottom of the nanorod array, representing the existence of a substrate and electrodes. Uniform force or stress is applied to the top electrode surface along the $z$-direction. One end is fixed and the other end is vibrated as the boundary conditions for the cantilever.

In the simulation for nanorod arrays, the thickness (height) $t$ and width $l$ of one nanorod are set to be 1 and $0.1 \mu \mathrm{m}$, respectively. The material is assumed to be (001)-orientated tetragonal $\mathrm{Pb}(\mathrm{Zr}, \mathrm{Ti}) \mathrm{O}_{3}$, whose properties are listed in Table 2 .

\section{Results and Discussion}

\subsection{Effect of aspect ratio of nanorods on output voltage}

Figure 4 shows the simulated dependence of the output voltage on the aspect ratio $t / l$ with constant stress applied to a single piezoelectric unit. The ordinate represents the voltage ratio of a film whose aspect ratio is sufficiently small (lower than $10^{-4}$ ). According to Eq. (2), the

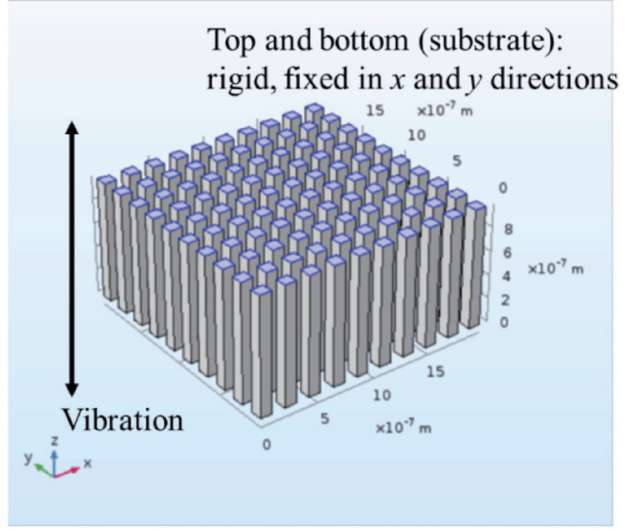

(a)

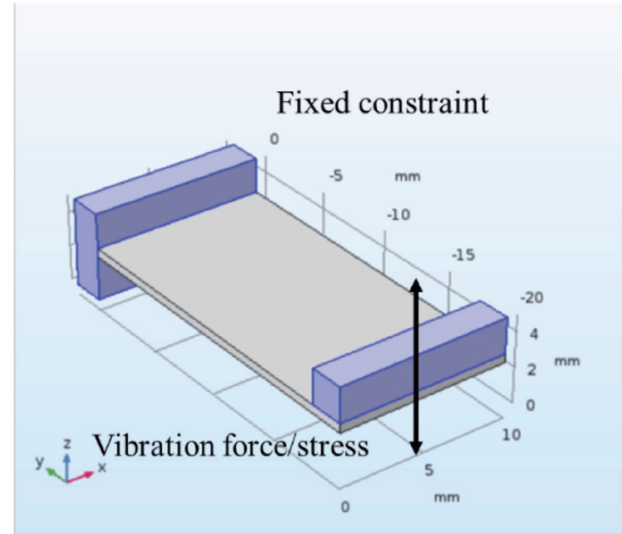

(b)

Fig. 3. (Color online) Boundary conditions for simulating the (a) nanorod array and (b) cantilever in COMSOL.

Table 2

Properties of $\mathrm{Pb}(\mathrm{Zr}, \mathrm{Ti}) \mathrm{O}_{3}$ used in simulation. ${ }^{(3,12)}$

\begin{tabular}{lcc}
\hline Property & Notation & Value \\
\hline \multirow{2}{*}{ Piezoelectric constants $(\mathrm{pC} / \mathrm{N})$} & $d_{33}$ & 133 \\
& $d_{31}$ & -45.8 \\
\hline \multirow{2}{*}{ Elastic compliance constants } & $s_{11}$ & 16.4 \\
$\left(10^{-12} \mathrm{~Pa}^{-1}\right)$ & $s_{12}$ & -5.74 \\
& $s_{13}$ & -7.22 \\
\hline Relative permittivity & $\varepsilon_{r}$ & 155 \\
\hline Density $\left(\mathrm{kg} \cdot \mathrm{cm}^{-3}\right)$ & $\rho$ & 7750 \\
\hline
\end{tabular}




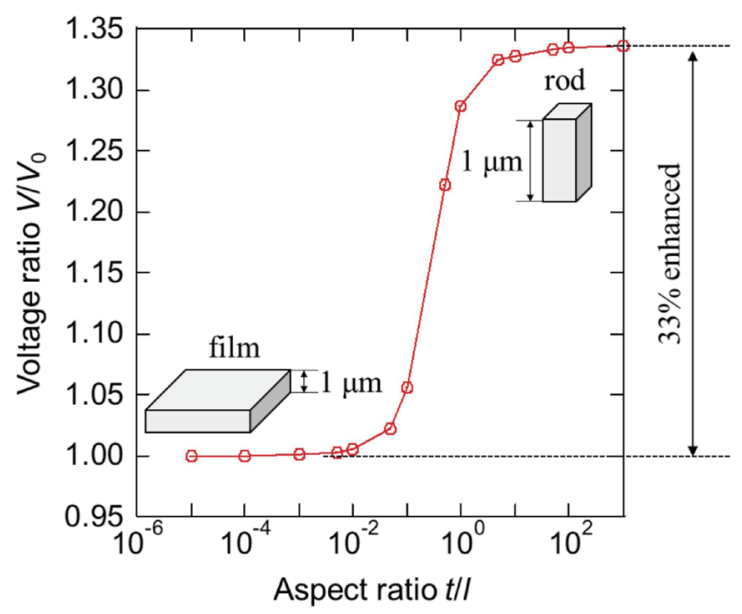

Fig. 4. (Color online) Simulated dependence of the output voltage on aspect ratio $t / l$ of a piezoelectric unit.

voltage is considered constant for the same applied stress and thickness unless $d_{33}$ varies. However, when the aspect ratio changes from 0.01 to 100 , the voltage was improved by $33 \%$, which indicates a $33 \% d_{33}$ enhancement and a $77 \% \mathrm{FOM}_{33}$ improvement of a piezoelectric unit. The enhancement of $d_{33}$ with a larger aspect ratio can be due to the reduced substrate clamping, which indeed well agrees with the prediction made using Eq. (5). In addition, a similar dependence has also been experimentally observed by Barzegar et al., ${ }^{(13)}$ who measured the $d_{33}$ of PZT ceramics with various aspect ratios. Although the material properties and size are different between our simulation and their experiment, the similar dependence supports our scenario.

To further reinforce our scenario, the deformation and stress map was constructed as shown in Fig. 5. The color of the surface represents the sum of the transversal stress. As seen in the figure, the rod-shaped model induces a lower transversal stress than the film model owing to less clamping by the substrates.

\subsection{Effect of area ratio of nanorods on output voltage and power}

In Sect. 3.1, the effect of the aspect ratio under the same applied stress is studied for a single nanorod unit. However, in the nanorod arrays, the contact area with substrates can vary with the density of nanorods; therefore, the stress applied to each nanorod will be larger for a structure with a lower density under the same vibration conditions. Since not only the aspect ratio but also the contact area can be simultaneously tuned by the condition of growth processes such as laser ablation, sputtering, and chemical vapor deposition, the effect of the area ratio on the output voltage and power can be one of the important optimization factors. Figure 6 shows the dependence of the output voltage and power on the area ratio of nanorods, $A / A_{0}$, as determined by calculation using Eqs. (6) and (8) as well as simulation. Here, $d_{33} / d_{33}^{\prime}$ of 1.3 based on Eq. (5) is adopted for the calculation. It is clear that both output voltage and power increase as the area ratio decreases because of the increased stress to nanorods. It is also worth 


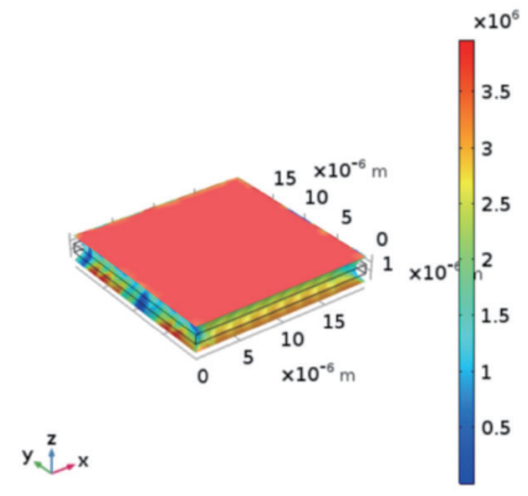

(a)

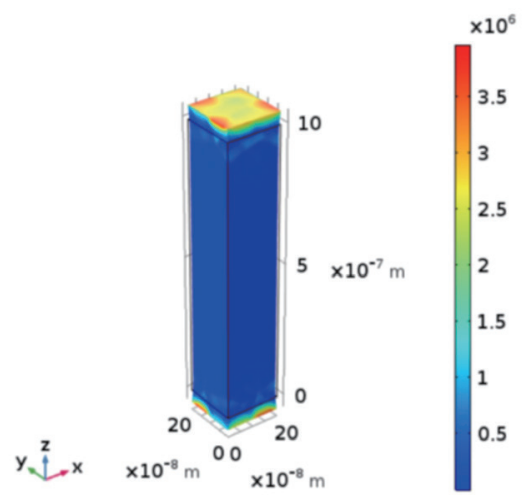

(b)

Fig. 5. (Color online) Deformation and transversal stress of (a) film and (b) rod.

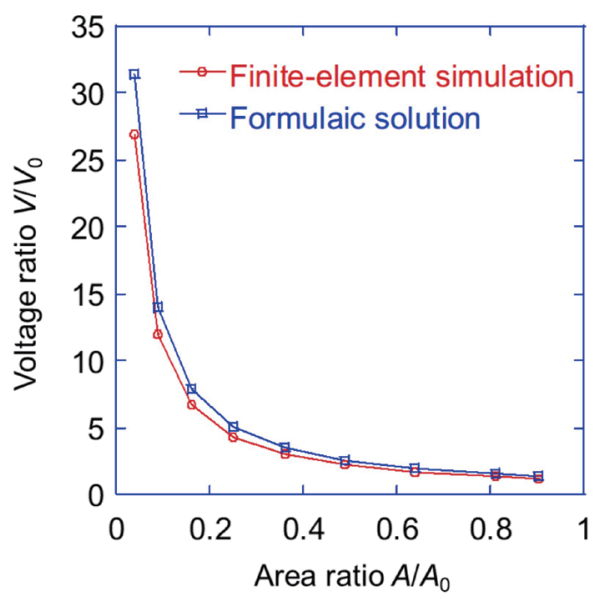

(a)

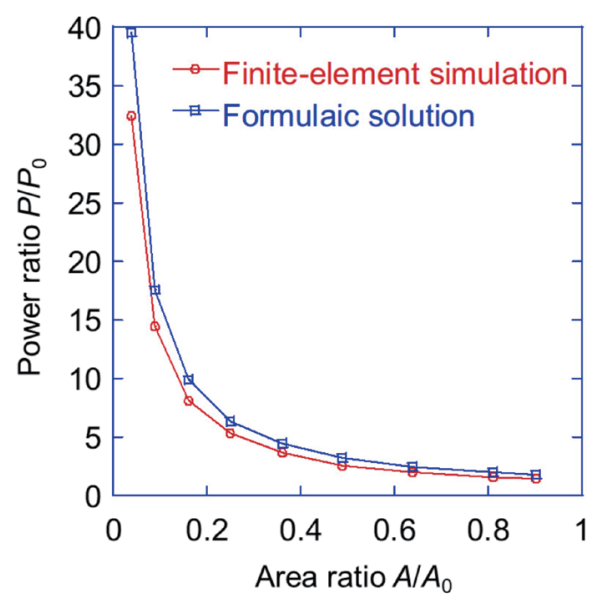

(b)

Fig. 6. (Color online) Dependence of the (a) output voltage and (b) power on the area ratio of nanorods.

noting that the output power can be one or more orders of magnitude higher than that of a film with the same volume under the same vibration conditions.

Therefore, the use of a nanorod structure is proved to be an effective way of improving the output power of the PEH device because of the enhanced $d_{33}$ (thus FOM) and stress of the piezoelectric materials. Since $d_{33}$ is always higher than $d_{31}$, the $d_{33}$ working mode will be more efficient as long as the generated stress is comparable to that of the $d_{31}$ mode used in a cantilever structure.

There are, however, several limitations for the practical use of nanorod arrays. For example, the enhancement of stress has a limitation because of the mechanical strength of the piezoelectric material. Since in the environment the vibration cannot be applied perfectly along the axial direction of the nanorods, it is inevitable that low-frequency lateral oscillations are generated. Such lateral oscillations cause shear stresses in the nanorods, which increase with decreasing area ratio of the nanorod array. Therefore, the nanogenerators have to be designed 
so that the induced stresses can be sufficiently below the fracture stress. It should also be noted that the output voltage and power in practical devices will be limited by mechanical damping and piezoelectric damping, although they are not major factors that limit power at off-resonance frequencies.

\subsection{Comparison with resonant cantilever}

A difficulty for the nanorod structure in the $d_{33}$ mode is to realize the resonance at vibration frequencies existing in the environment (lower than $300 \mathrm{~Hz}$ ) since the thickness excitation will only allow resonant frequencies of megahertz order or higher. Figure 7(a) shows the output power density of the nanorods and film in the $d_{33}$ mode, and of the conventional film cantilever structure in the $d_{31}$ mode with a resonant frequency of $105 \mathrm{~Hz}$. The piezoelectric layers in both structures are of the same volume, as illustrated in Fig. 7(c). The substrates and seismic mass are made of steel and the amplitude $F_{m}$ of vibration force for both devices is $100 \mu \mathrm{N}$. It is

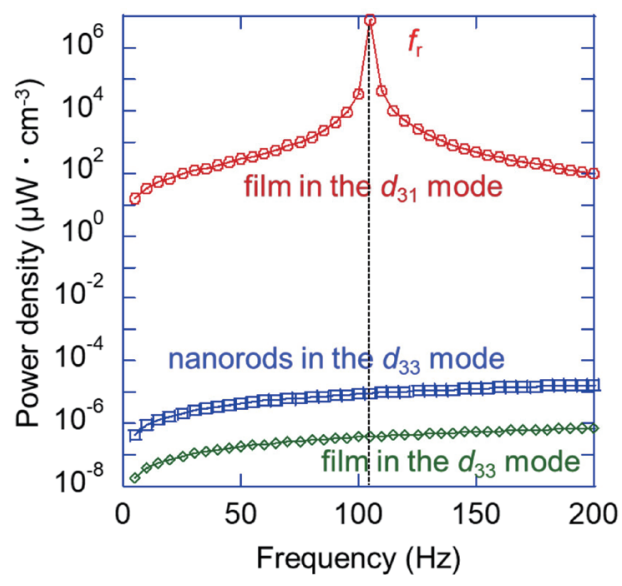

(a)

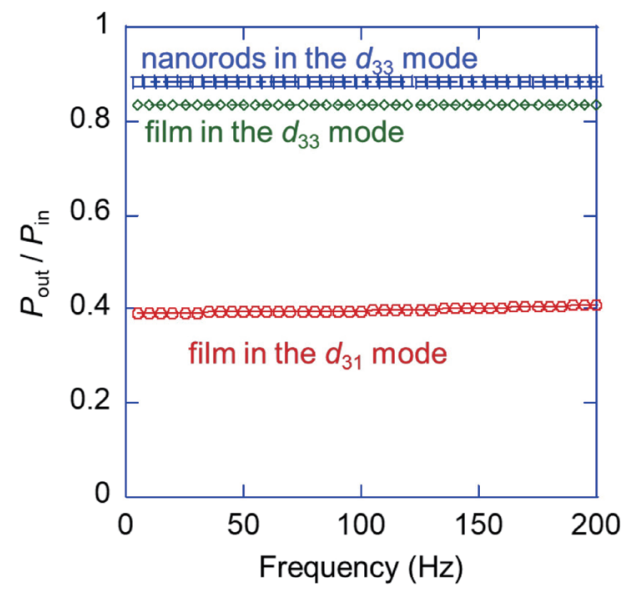

(b)

$t=1 \mu \mathrm{m}, t_{\text {sub }}=20 \mu \mathrm{m}, L=5 \mathrm{~mm}, W=1 \mathrm{~mm}, M=10 \mathrm{mg}$
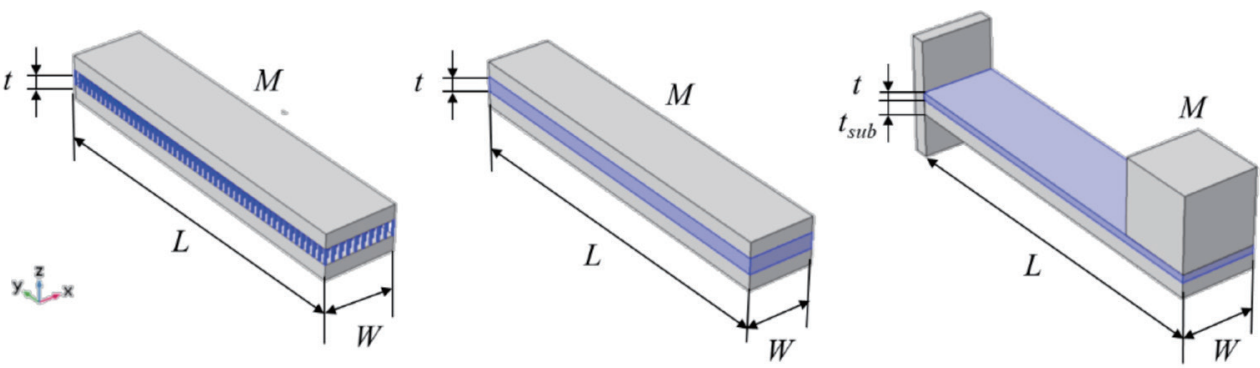

(c)

Fig. 7. (Color online) Output power among the nanorods and film in the $d_{33}$ mode, and the film cantilever in the $d_{31}$ mode. (a) Output power density under the same vibration force. (b) Ratio of output power to input power. (c) Schematic models for the nanorods and film in the $d_{33}$ mode, and the conventional film cantilever structure in the $d_{31}$ mode. 
clearly shown that the cantilever in the $d_{31}$ mode at resonance in particular has a much higher output than the $d_{33}$-mode structures. Equations (9) and (10) express the spring constant $k$ of the film or nanorods in the $d_{33}$ mode and that of the film cantilever in the $d_{31}$ mode, respectively.

$$
\begin{gathered}
k=\frac{L W Y}{t} \text { for film or nanorods in } d_{33} \text { mode } \\
k=\frac{W t_{s u b}{ }^{3} Y_{s u b}}{4 L^{3}} \text { for film cantilever in } d_{31} \text { mode }{ }^{(14)}
\end{gathered}
$$

Here, $Y$ and $Y_{\text {sub }}$ stand for Young's moduli of the piezoelectric and substrate, $W$ and $L$ for the width and length of the structure, respectively, and $t_{\text {sub }}$ for the thickness of the substrate. As $k$ in the $d_{33}$ mode is found to be larger than that in the $d_{31}$ mode, the cantilever can be more efficient for obtaining a higher input power than the $d_{33}$-mode film or nanorods with the same applied force. The input power was also simulated in COMSOL, which reflects the basic feature of $k$ for the $d_{33}$-mode film or nanorods and for the $d_{31}$-mode film cantilever. The conversion efficiency from input power to output power is plotted in Fig. 7(b). As can be seen in the figure, the conversion efficiency in the nanorods and film in the $d_{33}$ mode is higher than that in the cantilever.

Figure 8 shows the dependence of output power density at $100 \mathrm{~Hz}$ on the length of the device loaded with a constant mass. It is found that the output of the nanorods increases with decreasing device length and is expected to exceed that of the film cantilever in the $d_{31}$ mode when the length is less than $700 \mu \mathrm{m}$. This is because the resonant frequency of the film cantilever in the $d_{31}$ mode significantly deviates from the vibration frequency. It is obviously difficult to fabricate microscale cantilevers at such a low resonant frequency. Therefore, fabricating nanorods can be a good alternative for powering miniaturized microsensors.

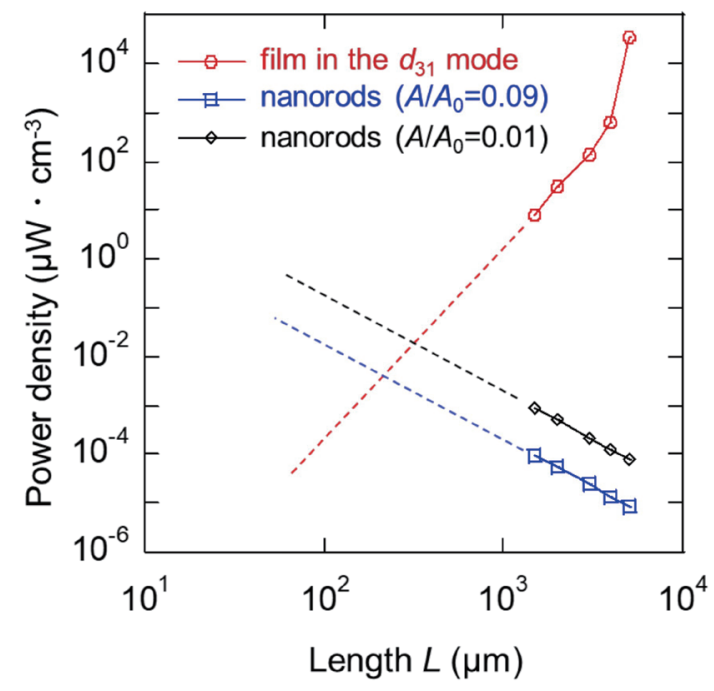

Fig. 8. (Color online) Dependence of output power density on the length of the device. 


\section{Conclusions}

As an effective method of improving the output power of PEH devices, an aligned nanorod array was considered in this study. For the same stress as that applied to a piezoelectric unit, the nanorod generated a $33 \%$ higher voltage than the film owing to the less mechanical clamping by the substrate. As the area ratio of nanorods decreased, the output power was one or more orders of magnitude higher than that of the film of the same volume under the same vibration conditions. These results indicate that the nanorod structure enhances not only FOM but also the stress, which may lead to practical use in off-resonant applications. In spite of the output power of the off-resonant nanorod array being far lower than that of the resonant cantilever, it can be widely used in powering miniaturized microsensors, where the resonance of a cantilever is difficult to achieve.

\section{Acknowledgments}

This work was mainly supported by JST-PRESTO "Scientific Innovation for Energy Harvesting Technology, Grant No. JPMJPR16R9".

\section{References}

1 F. K. Shaikh and S. Zeadally: Renew. Sust. Energ. Rev. 55 (2016) 1041. https://doi.org/10.1016/j.rser.2015.11.010

2 H. Sun, M. Yin, W. Wei, J. Li, H. Wang, and X. Jin: Microsyst. Technol. 24 (2018) 2853. https://doi. org/10.1007/s00542-018-3763-z

3 C. R. Bowen, H. A. Kim, P. M. Weaver, and S. Dunn: Energ. Environ. Sci. 7 (2014) 25. https://doi.org/10.1039/ C3EE42454E

4 A. Daniels, M. Zhu, and A. Tiwari: IEEE Trans. Ultrason. Ferroelectr. Freq. Control 60 (2013), 2626. https:// doi.org/10.1109/TUFFC.2013.2861

5 J. Song and J. Wang: Sci. China Technol. Sci. 59 (2016) 1012. https://doi.org/10.1007/s11431-016-6081-7

6 J. Zhao and Z. You: Sensors 14 (2014) 12497. https://doi.org/10.3390/s140712497

7 K. I. Park, M. Lee, Y. Liu, S. Moon, G. T. Hwang, G. Zhu, J. E. Kim, S. O. Kim, D. K. Kim, Z. L. Wang, and K. J. Lee: Adv. Mater. 24 (2012) 2999. https://doi.org/10.1002/adma.201200105

8 Y. Qin, X. Wang, and Z. L. Wang: Nature 451 (2008) 809. https://doi.org/10.1038/nature06601

9 L. Gu, N. Cui, L. Cheng, Q. Xu, S. Bai, M. Yuan, W. Wu, J. Liu, Y. Zhao, F. Ma, and Y. Qin: Nano Lett. 13 (2012) 91. https://doi.org/10.1021/n1303539c

10 T. Yamada, D. Ito, T. Sluka, O. Sakata, H. Tanaka, H. Funakubo, T. Namazu, N. Wakiya, M. Yoshino, T. Nagasaki, and N. Setter: Sci. Rep. 7 (2017) 5236. https://doi.org/10.1038/s41598-017-05475-x

11 K. Okamoto, T. Yamada, J. Yasumoto, K. Nakamura, M. Yoshino, and T. Nagasaki: J. Ceram. Soc. Jpn. 126 (2018) 276. https://doi.org/10.2109/jcersj2.17255

12 M. J. Haun, E. Furman, S. J. Jang, and L. E. Cross: Ferroelectrics 99 (1989) 13. https://doi. org/10.1080/00150198908221436

13 A. Barzegar, D. Damjanovic, and N. Setter: IEEE T. Ultrason. Ferr. 51 (2004) 262. https://doi.org/10.1109/ TUFFC.2004.1320781

14 E. L. Worthington: Piezoelectric Energy Harvesting: Enhancing Power Output by Device Optimisation and Circuit Techniques, PhD Thesis, Cranfield University (2010). http://dspace.lib.cranfield.ac.uk/handle/1826/4579 


\section{About the Authors}

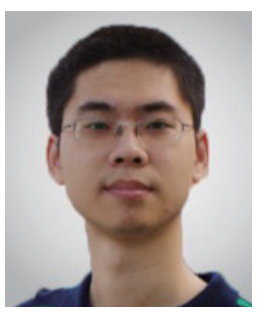

Jundong Song received his B.S. degree in materials forming and controlling engineering from Shandong University in 2014 and his M.Eng. degree in materials engineering from Tsinghua University in 2017. He is currently a Ph.D. student in the Department of Energy Engineering, Nagoya University. His current research interests include piezoelectric and ferroelectric thin films as well as their innovative applications.

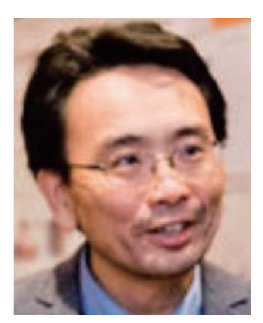

Tomoaki Yamada received his B.S. and Ph.D. degrees in metallurgy and ceramics science from the Tokyo Institute of Technology, Tokyo, Japan, in 1999 and 2003, respectively. He joined the Ceramics Laboratory, École Polytechnique Fédérale de Lausanne, Lausanne, Switzerland, in 2004. In 2008, he became an assistant professor at the Tokyo Institute of Technology under the Global COE Program. In 2010, he moved to Nagoya University, Nagoya, Japan, as an associate professor. Since 2010, he has held a concurrent researcher position in the JST-PRESTO program for developing novel piezoelectric nanostructures and energy harvesters. His current research interests include functional metal oxide thin films and nanostructures, and their devices.

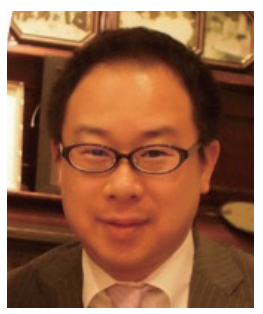

Masahito Yoshino received his B.S. and Ph.D. degrees in materials science and engineering from Nagoya University, Nagoya, Japan, in 1998 and 2003, respectively. He is currently an assistant professor at Nagoya University. His current research interests include the first-principles calculation, synthesis, and characterization of oxide materials as electronic and luminescent materials.

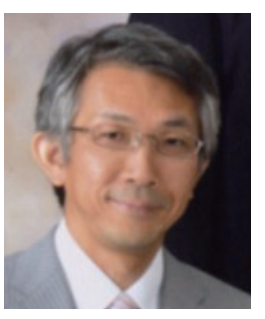

Takanori Nagasaki received his M.Eng. and D.Eng. degrees in nuclear engineering from the University of Tokyo, Tokyo, Japan, in 1983 and 1993, respectively. He was involved in the area of nuclear fusion materials with the Japan Atomic Energy Research Institute, Tokai-mura, Japan, from 1983 to 1995. In 1991, he was with Cambridge University, Cambridge, U.K., as a visiting scholar. Since 1995, he has been an associate professor and then a professor at Nagoya University, Nagoya, Japan. His current research interests include energy-related materials, especially materials to be used in a hydrogen energy system. 